\title{
Plastia uretral y calidad de vida asociada a la técnica quirúrgica en la Unidad Médica de Alta Especialidad Mérida
}

\author{
Urethral plasticity and quality of life associated with the surgical technique in Unidad \\ Médica de Alta Especialidad Mérida
}

\author{
Edgar Surur-Zaibaka, Patricia B. Bolado-García*, Gonzalo J. Gamboa-López, Tania Gómez-Ávalos y \\ Luis F. Mena-Ramírez \\ Unidad Médica de Alta Especialidad, Centro Médico Nacional Ignacio García Téllez, Instituto Mexicano del Seguro Social, Mérida, Yucatán, México
}

\begin{abstract}
Resumen
Objetivo: Determinar la calidad de vida asociada con la técnica quirúrgica en pacientes posoperados de plastia uretral. Método: Se estudiaron 29 pacientes intervenidos entre enero de 2011 y diciembre de 2015. Se aplicaron los cuestionarios Euroqol 5-D e International Prostate Symptom Score para medir calidad de vida y detectar recaídas, respectivamente. Se realizó estadística descriptiva, y se calcularon la incidencia acumulada, el riesgo relativo y la fracción atribuible. Resultados: $L a$ incidencia de recaídas detectada por el International Prostate Symptom Score fue del 69\% ( $p=0.011)$; el riesgo relativo fue de 2.19 (intervalo de confianza del 95\%: 2.092-2.288). La fracción atribuible a la exposición fue del 54\%. La calidad de vida se asoció con la longitud de la lesión $(p=0.046)$, los síntomas urinarios bajos $(p=0.004)$ y la percepción individual del estado de salud $(p=0.003)$. La localización de la lesión se asoció con recaída $(p=0.008)$. La calidad de vida no se asoció con el tipo de plastia ( $p$ > 0.05). Conclusiones: La incidencia de recurrencia posquirúrgica es alta. La calidad de vida es independiente de la técnica quirúrgica, pero está asociada con la longitud de la lesión y con los síntomas urinarios.
\end{abstract}

PALABRAS CLAVE: Plastia uretral. Calidad de vida. International Prostate Symptom Score. Euroqol 5-D. Recaída.

\begin{abstract}
Objective: To determine the quality of life associated with the surgical technique in postoperative patients with urethral plasty. Methods: 29 patients operated between January-2011 and December-2015 were studied. The questionnaires Euroqol 5-D and International Prostate Symptom Score were applied to measure quality of life and detect relapses, respectively. Descriptive statistics was performed, cumulative incidence, relative risk and attributable fraction were calculated. Results: the incidence of relapse detected by the International Prostate Symptom Score was 69\% ( $p=0.011)$; the relative risk was 2.19 (95\% confidence interval: $2.092-2.288)$. The fraction attributable to the exposure was $54 \%$. The quality of life was associated with the length of the lesion $(p=0.046)$, low urinary symptoms $(p=0.004)$ and the individual perception of the state of health $(p=0.003)$. The location of the lesion was associated with relapse $(p=0.008)$. Quality of life was not associated with type of plasty $(p>0.05)$. Conclusions: The incidence of postoperative recurrence is high. The quality of life is independent of the surgical technique, but it is associated with the length of the lesion and urinary symptoms.
\end{abstract}

KEY WORDS: Urethral plasty. Quality of life. International Prostate Symptom Score. Euroqol 5-D. Relapse.

\author{
Correspondencia: \\ *Patricia Berenice Bolado-García \\ CMD Calle 30x39 \#439 Col. Industrial, \\ Ex-terrenos El Fénix \\ C.P. 97150, Mérida, Yucatán, México \\ E-mail: investigacion.umae.imss@gmail.com; \\ patricia.bolado@imss.gob.mx
}

Fecha de recepción: 29-01-2018

Fecha de aceptación: 11-05-2018

DOI://dx.doi.org/10.24875/CIRU.M18000048
Cir Cir. 2018;86:313-320

Contents available at PubMed www.cirugiaycirujanos.com 


\section{Introducción}

«Estenosis uretral» es el término preferido para describir un estrechamiento anormal de cualquier segmento de la uretra rodeado por el cuerpo esponjoso, e implica específicamente grados variables de espongiofibrosis ${ }^{1}$. Es un padecimiento relativamente común en los hombres, con una frecuencia de 229$627 / 100,000$ hombres, y un $0.6 \%$ de la población en riesgo, generalmente hombres mayores ${ }^{2}$.

En la estenosis uretral se presenta síntomas y signos de obstrucción urinaria. Su etiología puede clasificarse como iatrogénica, traumática, inflamatoria e idiopática ${ }^{1}$. La plastia es una opción terapéutica cuyo propósito es una reconstrucción duradera y funcional. La recurrencia varía en función de la causa de la lesión, de los factores pronósticos, del tipo de unidad de atención médica y de la experiencia clínica y la destreza quirúrgica del médico tratante, además del riesgo implícito en el mismo procedimiento quirúrgico. Todos ellos serán los factores predisponentes al éxito $o$ al fracaso del tratamiento; sin embargo, la opinión o percepción del paciente en cuanto a la sensación de bienestar que puede experimentar y que representa la suma de sensaciones subjetivas y personales del "sentirse bien" ${ }^{3}$ debe ser evaluada con la finalidad de determinar el impacto de las intervenciones terapéuticas. La satisfacción de los usuarios y la calidad de vida relacionada con la salud deben convertirse en indicadores del desempeño de los sistemas de salud de los países, y tienen que ser inherentes a todas las especialidades médicas. La salud autopercibida se asocia de manera independiente con el diagnóstico clínico y permite predecir la utilización de los servicios de salud. Si los resultados de los cuidados para la salud no se miden desde la perspectiva de los pacientes, se puede considerar que la evaluación de las intervenciones es incompleta, ya que son los sujetos intervenidos quienes deben opinar sobre la forma en que sus vidas 0 su salud son afectadas. Para ello, recurrimos a instrumentos ya validados que miden la calidad de vida posterior a una asistencia en salud. Existen instrumentos genéricos cuyo objetivo es medir los cambios en la calidad de vida en diferentes momentos de la enfermedad. La determinación de las utilidades o preferencias que los individuos asignan a los diferentes estados de salud del instrumento proporciona una puntuación única que refleja numéricamente la calidad de vida (Euroqol 5-D e International Prostate Symptom Score [IPSS] $)^{3}$.

Por ello, el objetivo el presente trabajo fue determinar la calidad de vida asociada con la técnica quirúrgica en pacientes posoperados de plastia uretral, en un esfuerzo por contar con una mayor precisión de las consecuencias de la enfermedad y su tratamiento, y sobre todo de cómo el paciente se percibe después de un tratamiento, independientemente de sus condiciones físicas y del concepto de tratamiento exitoso de su médico tratante.

\section{Método}

Previa autorización del Comité Local de Investigación y Ética en Investigación en Salud N..$^{\circ} 3203$ de la Unidad Médica de Alta Especialidad (UMAE) Mérida, con número de registro R-2016-3203-7, se realizó un estudio observacional para determinar la calidad de vida en pacientes sometidos a plastia uretral entre enero de 2011 y diciembre de 2015. Se excluyeron pacientes con síntomas urinarios bajos no relacionados con estenosis uretral.

Se aplicaron los cuestionarios para medir la calidad de vida Euroqol 5-D e IPSS posterior a la plastia. EI grado de bienestar y satisfacción fue medido por la percepción del paciente asociado a la morbilidad inherente a la plastia, caracterizada por la recurrencia de la estenosis.

El octavo ítem del IPSS evaluó el impacto de los síntomas del tracto urinario bajo posteriores a la plastia como el grado de satisfacción de acuerdo con la percepción del paciente. Con el Euroqol 5-D se evaluaron cinco dimensiones de salud: movilidad, cuidado personal, actividades cotidianas, dolor/malestar y ansiedad/depresión; el dolor se evaluó por medio de una escala visual análoga.

Toda la información se concentró en una matriz de datos a la que solo tuvieron acceso los integrantes del grupo de investigación. La información se analizó con el programa estadístico SPSS v. 20.0 Se realizó estadística descriptiva. Se utilizaron las pruebas de ji al cuadrado y de Pearson. El grado de asociación de los factores pronósticos con la calidad de vida se realizó mediante el análisis de regresión logística y regresión lineal. Se calcularon la incidencia acumulada, el riesgo relativo con su intervalo de confianza del 95\% (IC 95\%) y la fracción atribuible a la exposición para la recurrencia. Se realizó una curva de Kaplan-Meier para analizar el tiempo libre de recurrencia de enfermedad. 


\section{Resultados}

Se estudiaron 29 pacientes posoperados de plastia uretral. El promedio de edad fue de $60.72 \pm 16.4$ años al momento de la reconstrucción quirúrgica. La media del índice de masa corporal fue de $27.99 \pm 3.92 \mathrm{~kg} / \mathrm{m}^{2}$. Se encontró diabetes mellitus tipo 2 en el $27.6 \%$ de los casos, e hipertensión arterial sistémica en el $44.8 \%$. De la totalidad de los casos, el $34.5 \%$ refirieron tabaquismo.

La causa iatrogénica se presentó en el $58.6 \%$ de los pacientes, seguida de la traumática en el $24.1 \%$, la idiopática en el $13.8 \%$ y la inflamatoria en el $4 \%$. La longitud media de la lesión uretral fue de $3.12 \pm$ $2.685 \mathrm{~cm}$; la moda de la longitud de la lesión fue $1 \mathrm{~cm}$ en el $27.6 \%$ de los pacientes $(p=0.44$; valor obtenido por tablas de contingencia, para los pacientes tratados con escisión y anastomosis).

La uretra bulbar estaba afectada en el $41.37 \%$ de los pacientes, seguida de la uretra peneana en el $20.6 \%$; el $37.93 \%$ de los pacientes tuvieron afectado más de un segmento uretral $(p=0.046$; valor obtenido por tablas de contingencia, para la totalidad del grupo). De los 29 pacientes, el $20.7 \%$ recibieron múltiples dilataciones uretrales previas a la plastia. Un $55.2 \%$ fueron tratados con uretrotomía interna bajo visión directa, de los cuales el $24.1 \%$ se sometieron al procedimiento en una ocasión, el $13.8 \%$ en dos, el $3.4 \%$ en tres, el $3.4 \%$ en cuatro y el $6.9 \%$ en cinco ocasiones; a un paciente se le realizaron 18 uretrotomías internas previas.

Respecto a la técnica quirúrgica empleada, al $55.2 \%$ se les realizó injerto de mucosa oral, y el $34.5 \%$ fueron tratados con escisión y anastomosis. Tres pacientes más fueron tratados con técnicas diferentes: uno fue intervenido con la técnica de Wolfe o injerto de piel retroauricular, con recaía a los 12 meses, y dos fueron tratados con más de una técnica quirúrgica y presentaron recaída a los 3 y 43 meses.

Con el análisis de regresión logística, la recaída se presentó en el $69 \%$ de los pacientes ( $p=0.047$; valor obtenido por análisis de regresión logística multivariada), y la certidumbre de predicción global del modelo fue del $89.7 \%$ ( $p=0.031$; valor obtenido por análisis de regresión logística multivariada). La distribución de los pacientes por etiología y por longitud de la lesión en quienes sufrieron recaída fue diferente según el tipo de plastia (tabla 1).

En cuanto a la presencia y gravedad de síntomas urinarios bajos de acuerdo con el IPSS, la distribución
Tabla 1. Porcentaje de recaída de los pacientes por etiología, longitud de la lesión y tipo de plastia realizada

\begin{tabular}{llcc}
\hline Tipo de plastia & Etiología de la & \multicolumn{2}{c}{ recaída* } \\
\cline { 3 - 4 } & Nosión & Sí \\
\hline $\begin{array}{l}\text { Escisión y anastomosis } \\
\text { primaria }\end{array}$ & Idiopática & 100 & 0 \\
& latrogénica & 20 & 80 \\
& Traumática & 75 & 25 \\
Injerto de mucosa oral & Idiopática & 0 & 100 \\
& Iatrogénica & 27.3 & 72.7 \\
& Traumática & 50 & 50 \\
& Inflamatoria & 0 & 100 \\
Injerto de Wolfe o de piel & Idiopática & 0 & 100 \\
retroauricular & & & \\
Más de una técnica quirúrgica & latrogénica & 0 & 100 \\
& Traumática & 0 & 100
\end{tabular}

Longitud de la lesión (cm)

Escisión y anastomosis

primaria

Injerto de mucosa oral

Injerto de Wolfe o de piel retroauricular

$\begin{array}{ccc}2 & 0 & 100 \\ 2 & 100 & 0\end{array}$

$\begin{array}{llll}\text { Más de una técnica quirúrgica } & 1 & 0 & 100\end{array}$

1

100

$2 \quad 0 \quad 100$

$3 \quad 0 \quad 100$

$3 \quad 40 \quad 60$

$\begin{array}{llll}4 & 50 \quad 50\end{array}$

$\begin{array}{llll}6 & 0 & 100\end{array}$

$10 \quad 0 \quad 100$

\begin{tabular}{lccc}
\hline & Mediana & \multicolumn{2}{c}{$\begin{array}{c}\text { Intervalo de } \\
\text { confianza del 95\% }\end{array}$} \\
\cline { 2 - 4 } & & $\begin{array}{c}\text { Límite } \\
\text { inferior }\end{array}$ & $\begin{array}{c}\text { Límite } \\
\text { superior }\end{array}$ \\
\hline $\begin{array}{l}\text { Escisión y anastomosis } \\
\text { primaria }\end{array}$ & 12 & 8.494 & 15.506 \\
$\begin{array}{l}\text { Injerto de mucosa oral } \\
\text { Injerto de Wolfe o de piel }\end{array}$ & 6 & 3.992 & 8.008 \\
retroauricular & 12 & - & - \\
Más de una técnica quirúrgica & 3 & - & - \\
\hline
\end{tabular}

*Los datos se expresan en porcentaje 


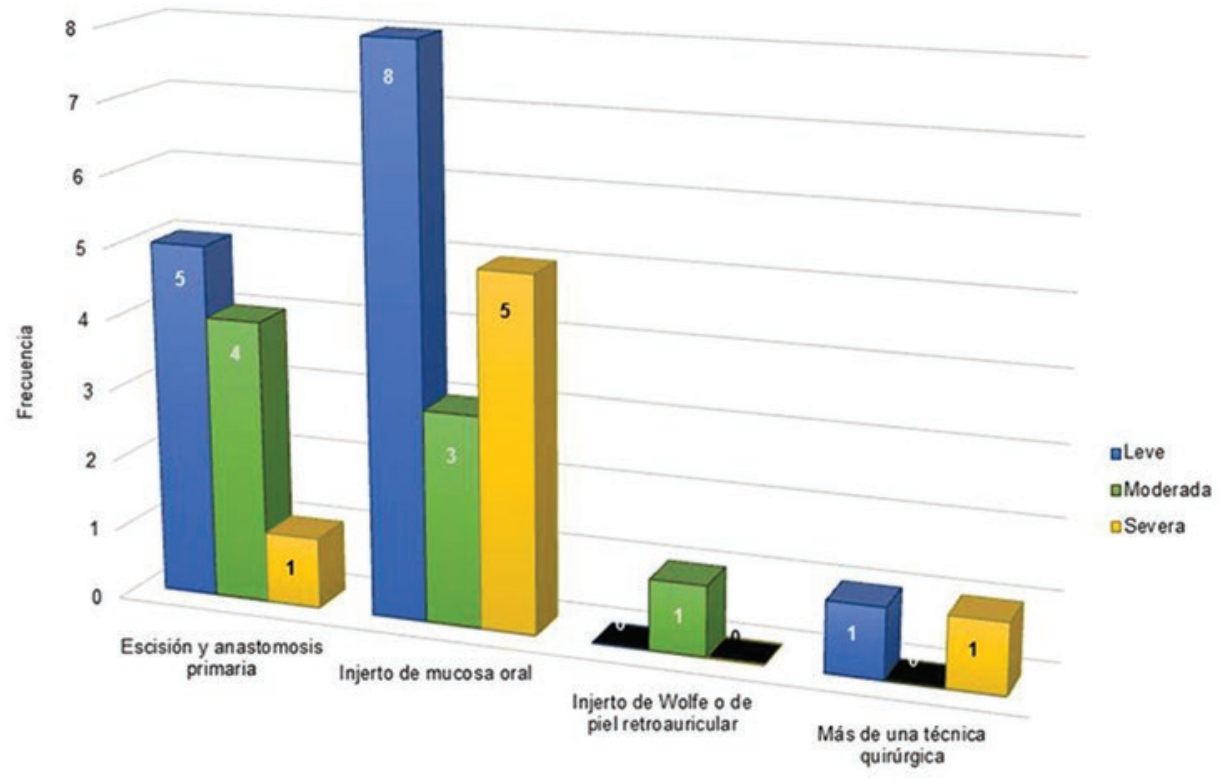

Figura 1. De acuerdo con la clasificación de la gravedad de los síntomas urinarios, la mayoría de los pacientes intervenidos con la técnica de injerto de mucosa oral presentaron síntomas urinarios leves, seguidos de síntomas graves. En este grupo se ubicaron más del $50 \%$ de los pacientes del estudio.

según la técnica quirúrgica se observa en la figura 1. La percepción de los pacientes de la calidad de vida después de la plastia de acuerdo con la respuesta al ítem 8 del IPSS se muestra en la figura 2. La distribución del estado de salud de acuerdo con el termómetro Euroqol de autovaloración del estado de salud se presenta en la figura 3. La percepción de los pacientes de la intensidad del dolor se representa en la figura 4.

La incidencia acumulada de recaída en la totalidad del grupo fue del $68 \%$. El riesgo relativo de presentar una recaída fue de 2.19 (IC 95\%: 1.69-2.8). La fracción atribuible a la exposición fue del $54 \%$. Los datos sobre el tiempo libre de recaída se muestran en la tabla 1. El riesgo absoluto de recaída para los pacientes posoperados con injerto de mucosa oral fue del 75\% (IC 95\%: 54-96). El riesgo absoluto de recaída para la técnica de escisión y anastomosis fue del $62 \%$ (IC 95\%: 35-88). El tiempo libre de recaída según el tipo de plastia se muestra en la figura 5 .

\section{Discusión}

El tratamiento de la estenosis uretral dependerá de la causa y de su mecanismo de lesión, así como de la longitud del defecto, el conocimiento y la experiencia del médico tratante para considerar la opción terapéutica más adecuada, y su habilidad quirúrgica para una reparación exitosa. Sin embargo, la percepción del paciente sobre sus condiciones de salud o enfermedad tras una intervención terapéutica es importante para determinar su grado de bienestar y satisfacción. Tomar en cuenta los factores específicos que pudieron influir para lograr o no una mejoría en la calidad de vida hace posible apoyar aspectos como la presencia de alguna complicación o discapacidad, o bien los problemas económicos relacionados con la propia enfermedad ${ }^{3}$. Los tratamientos urológicos se hacen cada vez más frecuentes conforme avanza la edad del paciente. La instrumentación de la uretra predispone a lesiones que requerirán un tratamiento posterior, cuya forma de presentación vendrá dada por las características de la población estudiada, el conocimiento y la experiencia clínica del médico tratante.

El pronóstico de los pacientes tratados por estenosis debe generar un interés académico entre especialistas y unidades médicas, y derivar en preguntas fundamentales sobre el proceso de atención médica, de las cuales la más básica es «¿Qué constituye un éxito quirúrgico?». Tradicionalmente, la definición académica de una uretroplastia exitosa es la falta de necesidad de un procedimiento secundario. Se ha demostrado que la estrechez asintomática de la luz uretral ocurre hasta en un 35\% de las estenosis recurrentes. De acuerdo con Erickson y Ghareeb ${ }^{4}$, el 


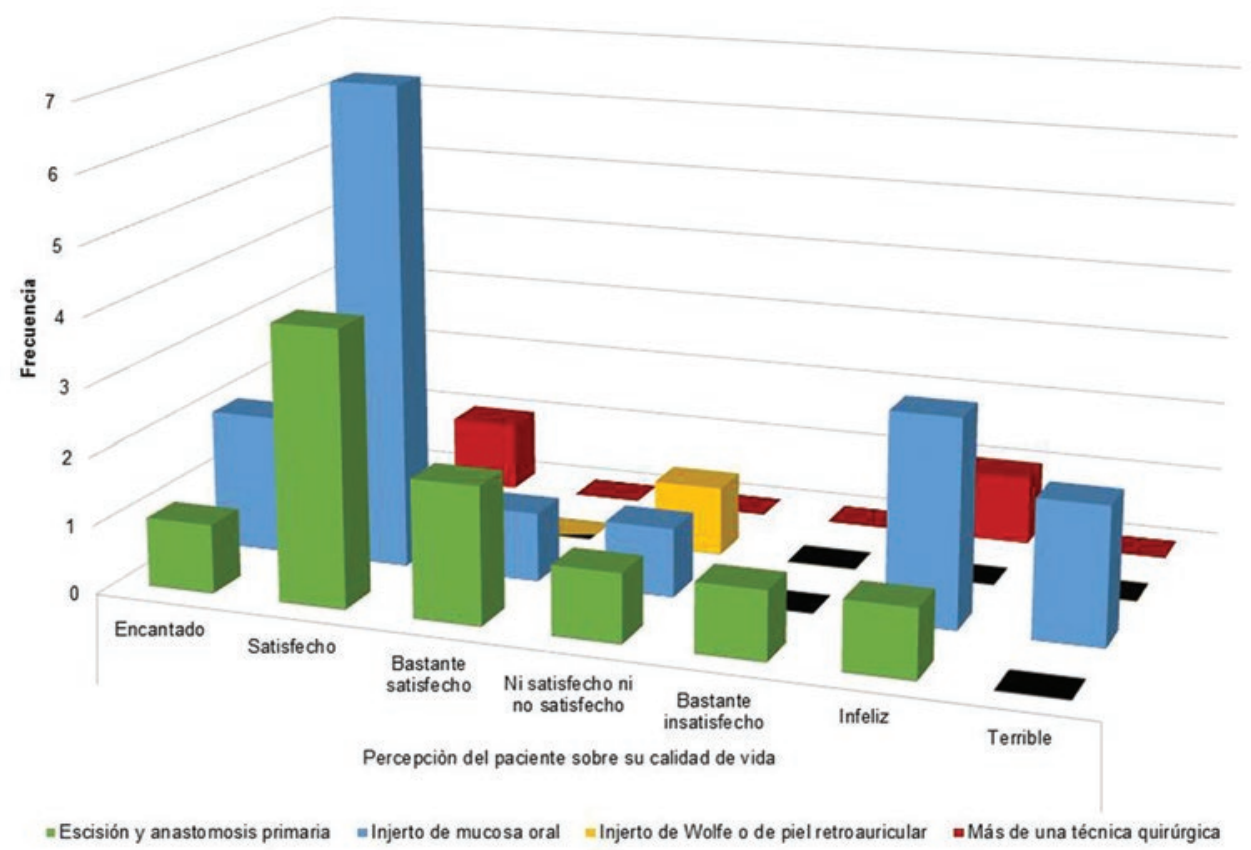

Figura 2. En general, de acuerdo con la respuesta recogida por el octavo ítem del IPSS, los pacientes refirieron estar satisfechos con su calidad de vida; algunos refirieron estar bastante satisfechos y otros la percibieron como excelente. Dos de tres pacientes en los que se realizó más de una técnica quirúrgica contestaron satisfecho y excelente. Esto pone de manifiesto la relevancia de tomar en consideración la opinión de los pacientes, independientemente del éxito de la técnica quirúrgica evaluado a través de la cistoscopia o de cualquier otro método de diagnóstico.

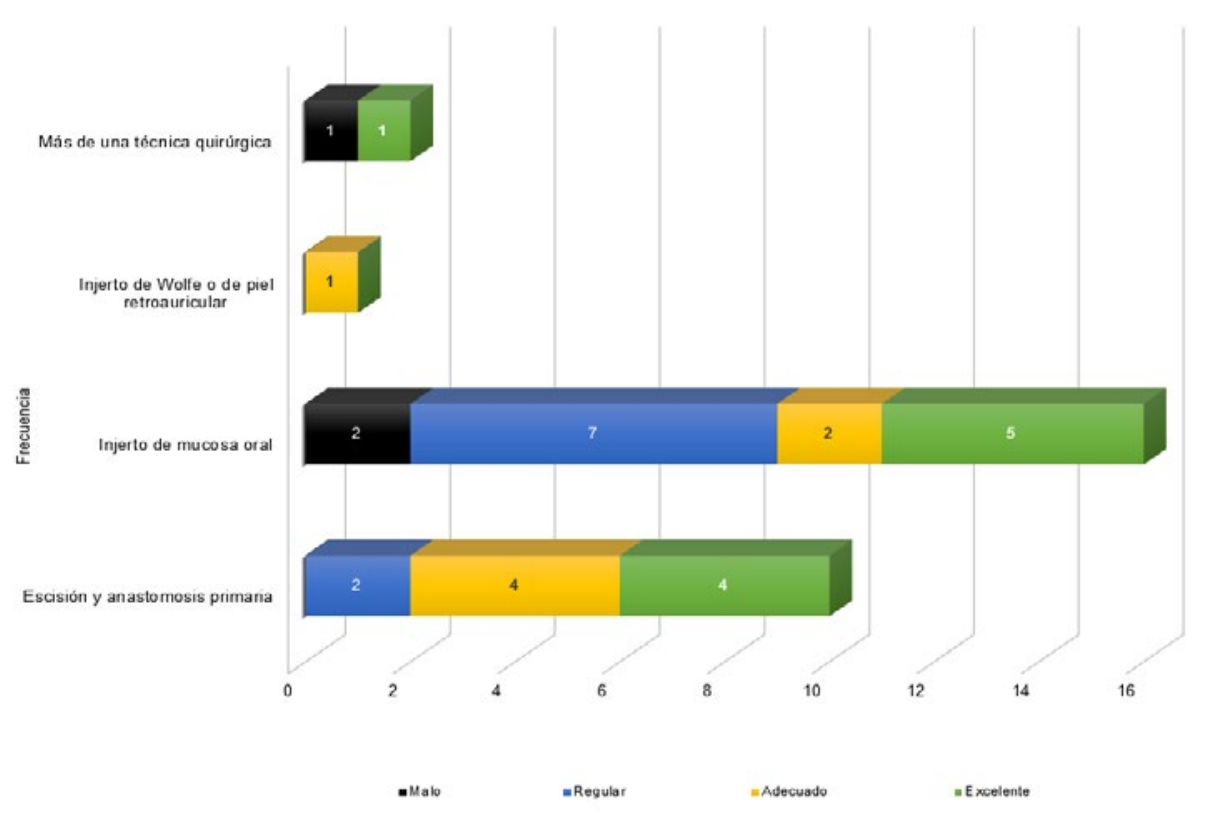

Figura 3. El estado de salud de los pacientes detectado a través del Termómetro EuroQol de Autovaloración del Estado de Salud, fue regular en la mayoría de los sujetos intervenidos con la técnica de injerto de mucosa oral. Sin embargo, los pacientes operados con escisión y anastomosis refirieron un estado de salud adecuado o excelente. A pesar de la diferencia de sujetos en cada grupo, esto permite visualizar que, según la técnica quirúrgica, el paciente se siente mejor con la de escisión y anastomosis, lo que puede ser un punto de referencia para trabajos futuros en los que puedan compararse ambas técnicas.

éxito quirúrgico después de una uretroplastia se relaciona con la complejidad anatómica de la técnica reconstructiva. Bertrand, et al..$^{5}$ reportaron que los hombres con cistoscopia recurrente tienen más probabilidades de estar insatisfechos (odds ratio: 4.96; IC 95\%: 2.07-11.90) y tienen medidas de uroflujometría significativamente peores; el $89.38 \%$ de los pacientes que estudiaron refirieron estar satisfechos 


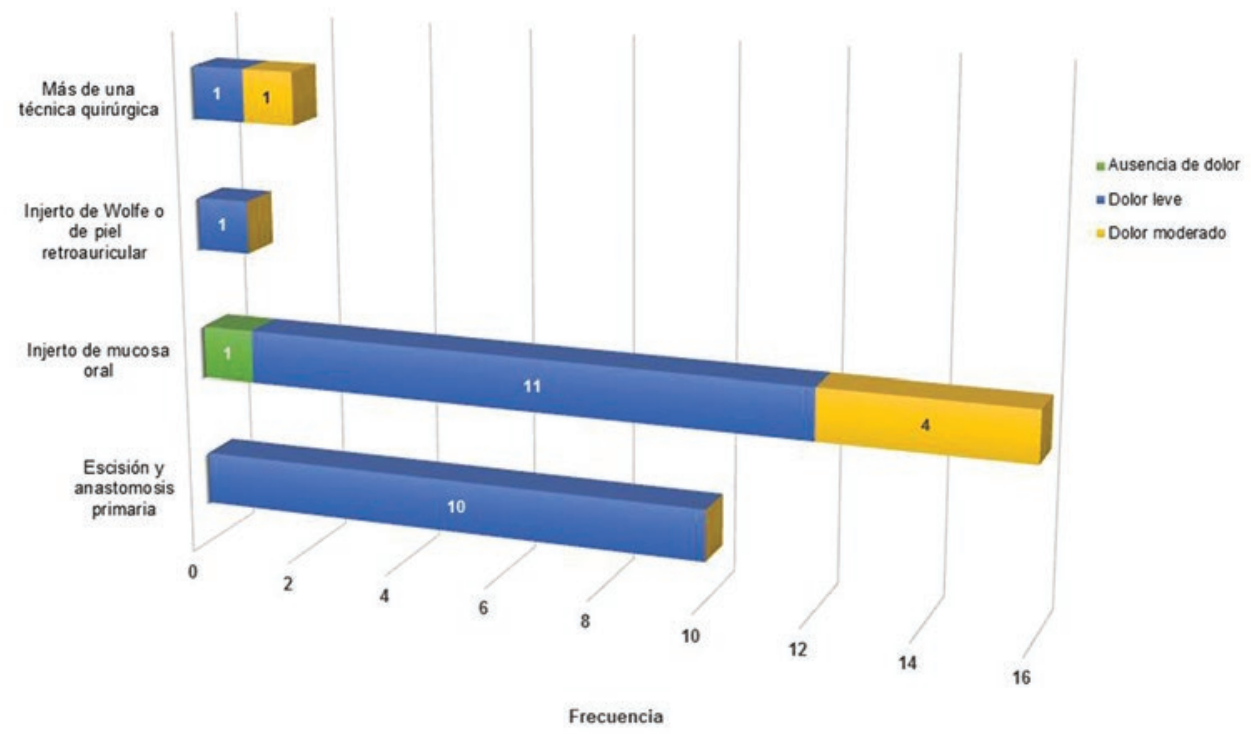

Figura 4. La medición del dolor a través de la escala visual análoga dejó de manifiesto que los pacientes con injerto pueden tener dolor posterior a la plastia. La técnica con escisión y anastomosis produjo mínima sensación dolorosa en ese grupo de pacientes. Estos datos pueden ser la pauta para estudios futuros o para normar protocolos de manejo para los pacientes con estenosis de uretra.

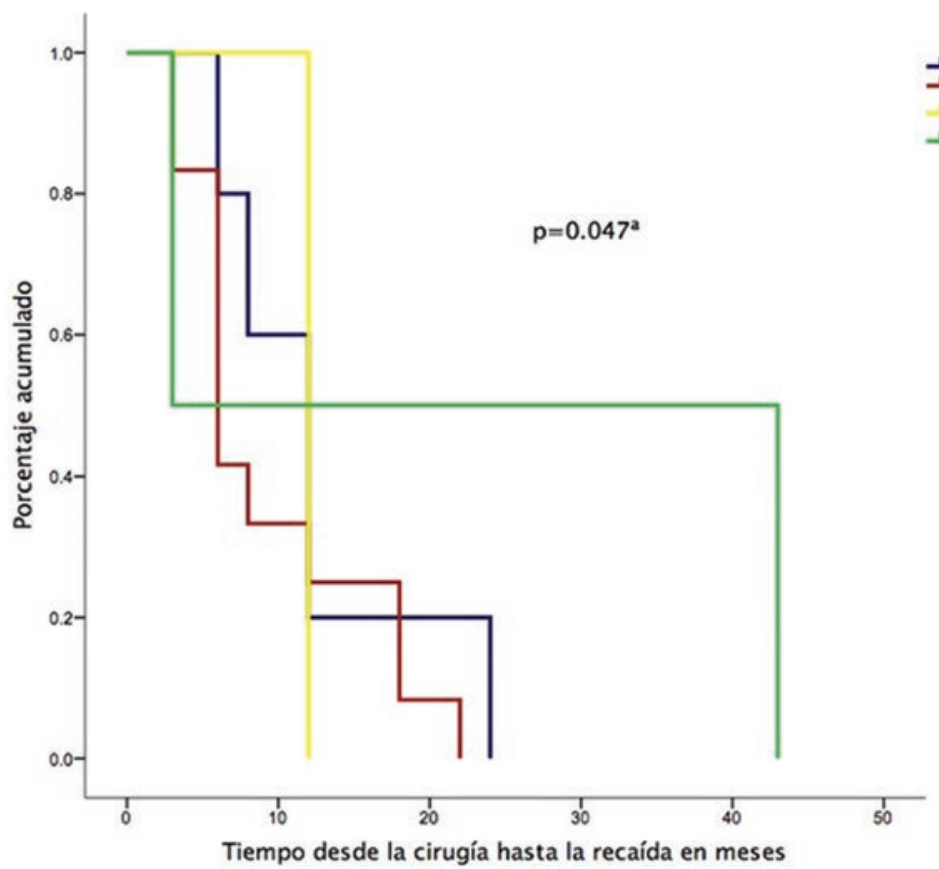

Figura 5. El tiempo libre de recaída con cada tipo de intervención quirúrgica fue variable; sin embargo, la mayoría de los sujetos presentaron recaída entre los 6 y los 12 meses. A pesar de la variabilidad en el tiempo libre de recaída, las dos técnicas quirúrgicas más frecuentes presentaron el 100\% de recaídas entre los 20 y 25 meses. Aunque dos pacientes requirieron plastia con más de una técnica quirúrgica, la recaída fue más tardía y se presentó hasta 43 meses después de la operación. El análisis de regresión resultó con significancia estadística importante, que complementó lo mostrado por el análisis de Kaplan-Meier.

o muy satisfechos después de ser intervenidos de plastia anterior, y aquellos que reportaron pocos síntomas urinarios bajos y menos dolor según el IPSS refirieron una mayor satisfacción.

Otra pregunta asociada debe ser cómo definir el de monitorear el paciente posuretroplastia?». La mayoría de los pacientes solo se preocupan por su capacidad para vaciar la vejiga. La estrategia de seguimiento ideal debe ser capaz de dar cuenta tanto del cirujano como de los pacientes: considerar la capacidad del cirujano para definir el éxito o el fracaso, 
y proteger la salud genitourinaria de los pacientes sin someterlos a procedimientos innecesarios. De acuerdo con el estudio de Liu, et al. ${ }^{6}$, la mitad de las recidivas se presentan en el plazo de 1 año, y la mayoría se manifiesta dentro de los primeros 6 meses. En los pacientes intervenidos en la UMAE Mérida se encontró una recurrencia del $69 \%$, con un promedio de 8 meses para hacerse presente. Los pacientes con lesiones uretrales cortas presentaron menor recurrencia y percepción de mejor estado de salud. La duración y la intensidad de la vigilancia después de la uretroplastia deben ser individualizadas. Es importante poder definir a los pacientes con alto riesgo de recurrencia y en qué periodo de tiempo, basándose en las características del paciente, la enfermedad y la técnica reconstructiva, permitiendo el diseño de protocolos de seguimiento individualizados ${ }^{6}$. Parece razonable utilizar estrategias validadas para medir resultados clínicos, evaluar los síntomas urinarios antes y después de la cirugía, y poder detectar la recurrencia, ya que se espera que la mayoría de los pacientes presenten síntomas nuevos o persistentes de la micción ${ }^{4}$. La aplicación del Euroqol-5D y del IPSS nos permitió encontrar una recaída en más de la mitad de los casos. Con estos instrumentos, el grado de bienestar y satisfacción fue medido por la percepción del paciente asociado con la morbilidad inherente a la plastia, caracterizada por la recurrencia de la estenosis. Con el IPSS, la recurrencia se determinó por la presencia de síntomas del tracto urinario bajo (intermitencia del flujo urinario, esfuerzo o pujo para iniciar la micción, flujo débil, frecuencia urinaria, nicturia, urgencia, sensación de vaciado incompleto) y la calidad de vida. La mayoría de los pacientes refirieron sentirse entre bastante satisfechos y encantados. Sin embargo, Tam, et al. ${ }^{7}$ realizaron un estudio para validar el IPSS y reportaron que el área bajo la curva ROC de la pregunta sobre la calidad de vida fue de 0.66 , y que la totalidad del instrumento fue de 0.56 . Existen diferencias con nuestros pacientes que deben ser consideradas y debe manejarse la información con precaución. La respuesta a la pregunta sobre la calidad de vida depende de la percepción del paciente. Los sujetos estudiados por Tam, et al. ${ }^{7}$ fueron en promedio de 45 años, más jóvenes que los nuestros, y por lo tanto el concepto de satisfacción puede ser diferente. En ese estudio, la comparación del IPSS se hizo contra la cistoscopia y no se refiere de qué manera este procedimiento invasivo permite medir la calidad de vida de un paciente con o sin recaída de la estenosis; la integridad de la reparación quirúrgica se evaluó a los 3, 6 y 12 meses.
Los pacientes que estudiamos fueron seguidos hasta los 43 meses de la intervención, y la mayoría de los casos que recayeron lo hicieron durante el primer año tras la plastia. Si bien es cierto que la cistoscopia permite visualizar el éxito del procedimiento quirúrgico en cuanto a evidencia física sobre su integridad, no explica la percepción del paciente de su estado de salud. Los instrumentos clínicos como los cuestionarios, en los que los puntajes dependen de la respuesta subjetiva del paciente, deben ser comparados con similares, ya que corremos el riesgo de subestimar una herramienta que puede ser de utilidad. Para ello, debemos tener claro el alcance de nuestras intervenciones y qué esperamos de ellas para determinar de qué manera serán evaluadas. La satisfacción del paciente después de una plastia uretral no se debe únicamente a la disminución de las molestias urinarias, sino también a la ausencia de dolor. Dado lo importante, aunque a menudo discordante, tanto de los hallazgos anatómicos como de los informados por el paciente que determinan el éxito después de la uretroplastia, ambos parámetros deben ser evaluados de manera simultánea, pero reportados por separado para todas las intervenciones. Esto facilitará la comparación de los resultados quirúrgicos entre diferentes cirugías, distintos cirujanos y diversos tipos de estenosis ${ }^{4}$.

Además de la evaluación de la recaída posterior a la cirugía y cómo percibe el paciente su estado de salud, es necesario indagar sobre el impacto de la intervención en la vida del paciente. Para ello se cuenta con otro instrumento genérico validado, el Euroqol 5-D, que evalúa cinco dimensiones de salud: movilidad, cuidado personal, actividades cotidianas, dolor/malestar y ansiedad/depresión ${ }^{8}$. Jackson, et al. ${ }^{9}$ utilizaron este instrumento para evaluar la reconstrucción de la uretra desde la perspectiva del paciente. Encontraron que el $33 \%$ de los pacientes estuvieron satisfechos y el $54 \%$ muy satisfechos. Nuestros pacientes intervenidos con injerto de mucosa oral refirieron, en su mayoría, un estado de salud regular (43.75\%), en tanto que el $31.25 \%$ refirió un excelente estado de salud, dejando ver que una técnica tiene mejores resultados que la otra. En contraste, los estados de salud predominantes con la técnica de escisión y anastomosis fueron adecuado y excelente. Sin embargo, son datos que deben ser tomados con reserva, ya que el número de sujetos intervenidos con cada técnica fue diferente (16 y 10, respectivamente). Aunado a esto hallamos una asociación significativa entre la longitud de la lesión y la percepción que el 
paciente tiene de su estado de salud a través del EuroQol-5D. Aunque el objetivo de los instrumentos utilizados es medir la calidad de vida, difieren en sus ítems. A pesar de ello, encontramos que la presencia de síntomas urinarios bajos detectados por medio del IPSS tuvo una asociación significativa con la percepción del paciente sobre su estado de salud a través del EuroQol-5D ( $p=0.004$ ); cuantos más síntomas refirieron los pacientes, mayor fue el deterioro de la percepción de su estado de salud. Además, la percepción individual del estado de salud de cada paciente evaluada con la octava pregunta del IPSS se relacionó significativamente con la calidad de vida evaluada en el EuroQol-5D ( $p=0.003)$. Estos datos nos permiten considerar que, al menos para esta población estudiada, la información recabada por uno u otro instrumento nos orienta sobre la percepción que el paciente tiene de su condición clínica. La realización de la uretroplastia vale la pena desde la perspectiva de los pacientes. El uso de instrumentos validados para medir la calidad de vida puede servir como punto de referencia con el cual los cirujanos y las instituciones médicas pueden comparar su desempeño ${ }^{9}$. La información que proporcionan permite identificar distintos estados de morbilidad y bienestar, y evaluar el impacto de las intervenciones terapéuticas y sanitarias en los individuos.

\section{Conclusiones}

La probabilidad de recaer después de la plastia es 2.19 veces mayor. La incidencia acumulada fue del $68 \%$ y la fracción atribuible a la exposición al procedimiento quirúrgico fue del $54 \%$. Es necesario generar bases de datos que permitan observar el comportamiento del fenómeno a través del tiempo y en la población atendida, y que incluyan la medición de cómo el paciente se percibe a sí mismo y a su entorno.

Existen limitaciones al estudio que deben ser reconocidas. El tamaño de la muestra, a pesar de representar el universo de pacientes del hospital, no es suficiente para permitir un análisis detallado de los diferentes subgrupos. La mayoría de los pacientes tienen un nivel sociocultural limitado, lo que dificulta la lectura, la compresión y el llenado del cuestionario. A pesar de ello, es importante recalcar que supone una ventaja que el mismo cirujano haya operado a todos los pacientes estudiados, lo que disminuye las variaciones en las técnicas quirúrgicas, pero al mismo tiempo representa también una limitante para comparar diversos criterios médicos. A pesar de sus limitaciones, el estudio es uno de los pocos realizados en México que evalúa la calidad de vida en función de la cirugía reconstructiva elegida.

El éxito del tratamiento de la estenosis de uretra deberá integrarse con la percepción que el paciente tiene de su calidad de vida y de su estado de salud, además de la técnica quirúrgica empleada y de las condiciones del paciente.

\section{Financiación}

El presente trabajo no ha recibido financiación alguna.

\section{Conflicto de intereses}

Los autores declaran no tener conflicto de intereses.

\section{Bibliografía}

1. Jordan G, Chapple C, Heyns C. An international consultation on urethral strictures. Marrakech, Morocco; 2012. Disponible en: www.siu-urology. org

2. Alwaal A, Blaschko SD, McAninch JW, Breyer BN. Epidemiology of urethral strictures. Transl Androl Urol. 2014;3:209-13.

3. Moreno Altamirano L. Epidemiología clínica. 3. ${ }^{a}$ ed. Ciudad de México: McGraw-Hill; 2013. 329 p.

4. Erickson BA, Ghareeb GM. Definition of successful treatment and optimal follow-up after urethral reconstruction for urethral stricture disease. Urol Clin North Am. 2017;44:1-9.

5. Bertrand LA, Voelzke BB, Elliott SP, Myers JB, Breyer BN, Vanni AJ, et al. Measuring and predicting patient dissatisfaction after anterior urethroplasty using patient reported outcomes measures. J Urol. 2016;196:453-61.

6. Liu JS, Dong C, González CM. Risk factors and timing of early stricture recurrence after urethroplasty. Urology. 2016;95:202-7.

7. Tam CA, Elliott SP, Voelzke BB, Myers JB, Vanni AJ, Breyer BN, et al. The International Prostate Symptom Score (IPSS) is an inadequate tool to screen for urethral stricture recurrence after anterior urethroplasty. Urology. 2016;95:197-201.

8. Dolan P. Modeling valuations for EuroQol health states. Med Care. 1997;35:1095-108.

9. Jackson MJ, Chaudhury I, Mangera A, Brett A, Watkin N, Chapple CR, et al. A prospective patient-centred evaluation of urethroplasty for anterior urethral stricture using a validated patient-reported outcome measure. Eur Urol. 2013;64:777-82. 